\title{
Article
}

\section{Influence of Including Patients with Premorbid Disability in Acute Stroke Trials: The HeadPoST Experience}

Wang, Xia, Moullaali, Tom J, Ouyang, Menglu, Billot, Laurent, Sandset, Else Charlotte, Song, Lili, Delcourt, Candice, Hackett, Maree, Watkins, Caroline Leigh, Robinson, Thompson G, Yang, Jie, Lavados, Pablo M, Brunser, Alejandro, Olavarría, Verónica V, Muñoz-Venturelli, Paula, Arima, Hisatomi, Middleton, Sandy, Pontes-Neto, Octávio M, Pandian, Jeyaraj Durai, Rogers, Kris and Anderson, Craig S

Available at http://clok.uclan.ac.uk/36499/

Wang, Xia, Moullaali, Tom J, Ouyang, Menglu, Billot, Laurent, Sandset, Else Charlotte, Song, Lili, Delcourt, Candice, Hackett, Maree ORCID: 0000-00031211-9087, Watkins, Caroline Leigh ORCID: 0000-0002-9403-3772 et al (2021) Influence of Including Patients with Premorbid Disability in Acute Stroke Trials: The HeadPoST Experience. Cerebrovascular diseases . ISSN $1015-9770$

It is advisable to refer to the publisher's version if you intend to cite from the work. http://dx.doi.org/10.1159/000512608

For more information about UCLan's research in this area go to http://www.uclan.ac.uk/researchgroups/ and search for <name of research Group>.

For information about Research generally at UCLan please go to http://www.uclan.ac.uk/research/

All outputs in CLoK are protected by Intellectual Property Rights law, including Copyright law. Copyright, IPR and Moral Rights for the works on this site are retained by the individual authors and/or other copyright owners. Terms and conditions for use of this material are defined in the policies page. 
Influence of including patients with pre-morbid disability in acute stroke trials: the

\section{HeadPoST experience}

Xia Wang, ${ }^{1,2}$ Tom J Moullaali, ${ }^{3}$ Menglu Ouyang, ${ }^{1}$ Laurent Billot, ${ }^{1}$ Else Charlotte Sandset, ${ }^{4,5}$ Lili Song, ${ }^{6}$ Candice Delcourt, ${ }^{1,7,8}$ Maree L. Hackett, ${ }^{1,9}$ Caroline L Watkins, ${ }^{9}$ Thompson G. Robinson, ${ }^{10}$ Jie Yang, ${ }^{2}$ Pablo M. Lavados, ${ }^{11}$ Alejandro Brunser, ${ }^{11}$ Veronica V. Olavarria, ${ }^{11}$ Paula Munoz Venturelli, ${ }^{11,12}$ Hisatomi Arima, ${ }^{13}$ Sandy Middleton, ${ }^{14}$ Octávio M. Pontes-Neto, ${ }^{15}$ Jeyaraj Durai Pandian, ${ }^{16}$ Kris Rogers, ${ }^{1}$ Craig S. Anderson; $;, 5,7,12$ on behalf of the HeadPoST Investigators

${ }^{1}$ The George Institute for Global Health, Faculty of Medicine, University of New South Wales, NSW, Australia

${ }^{2}$ Department of Neurology, the First Affiliated Hospital of Chengdu Medical College, Chengdu, China

${ }^{3}$ Centre for Clinical Brain Sciences, University of Edinburgh, Edinburgh, UK

${ }^{4}$ Department of Neurology, Oslo University Hospital, Oslo, Norway

${ }^{5}$ The Norwegian Air Ambulance Foundation, Oslo, Norway

${ }^{6}$ The George Institute China at Peking University Health Science Center, Beijing, PR China

${ }^{7}$ The University of Sydney, Sydney, Australia

${ }^{8}$ Neurology Department, Royal Prince Alfred Hospital, Sydney, NSW, Australia

${ }^{9}$ Faculty of Health and Care, University of Central Lancashire, Preston, Lancashire

${ }^{10}$ University of Leicester, Department of Cardiovascular Sciences and NIHR Leicester

Biomedical Research Centre, Leicester, UK

${ }^{11}$ Unidad de Neurología Vascular, Servicio de Neurología, Departamento de Neurologíay Psiquiatría, Clínica Alemana de Santiago, Facultad de Medicina, Universidad del Desarrollo, Santiago, Chile

${ }^{12}$ Departamento de Ciencias Neurológicas, Facultad de Medicina, Universidad de Chile, Santiago, Chile

${ }^{13}$ Department of Public Health, Fukuoka University, Japan

${ }^{14}$ Nursing Research Institute, Australian Catholic University and St Vincent's Health Network Sydney

${ }^{15}$ Ribeirão Preto Medical School, University of São Paulo, Ribeirão Preto, Brazil

${ }^{16}$ Department of Neurology, Christian Medical College, Ludhiana, Punjab, India 141008

\section{Author for correspondence:}

Professor Craig Anderson

The George Institute for Global Health, PO Box M201, Missenden Rd, NSW 2050, Australia T: +61-2-9993-4500, F: +61-2-9993-4502, E: canderson@georgeinstitute.org.au

Short title: Early post-stroke disability and outcomes

Word count: body 2648; abstract 250 


\begin{abstract}
Background: Patients with pre-morbid functional impairment are generally excluded from acute stroke trials. We aimed to determine the impact of including such patients in the Head Positioning in acute Stroke Trial (HeadPoST) and early additional impairment on outcomes.

Methods: Post-hoc analyses of HeadPoST, an international, cluster crossover randomized trial of lying-flat versus sitting-up head positioning in acute stroke. Associations of early additional impairment, defined as change in modified Rankin scale (mRS) scores from premorbid levels (estimated at baseline) to Day 7 ('early $\Delta \mathrm{mRS}$ '), and poor outcome (mRS score 3-6) at Day 90, were determined with generalized linear mixed model. Heterogeneity of the trial treatment effect was tested according to pre-morbid mRS scores 0-1 versus 2-5.
\end{abstract}

Results: Of 8,285 patients ( $38.9 \%$ female, mean age $68 \pm 13$ years) with complete data, there were 1,984 (23.9\%) with pre-morbid functional impairment (mRS 2-5). A significant linear association was evident for early $\Delta \mathrm{mRS}$ and poor outcome (per 1-point increase in $\Delta \mathrm{mRS}$, adjusted odds ratio 1.20, 95\% confidence interval 1.14-1.27; P <0.0001). Patients with greater pre-morbid functional impairment were less likely to develop additional impairment, but their risk of poor 90-day outcome significantly increased with increasing (worse) premorbid $\mathrm{mRS}$ scores (linear trend $\mathrm{P}<0.0001$ ). There was no heterogeneity of the trial treatment effect by level of pre-morbid function.

Conclusions: Early post-stroke functional impairment that exceeded pre-morbid levels was associated with worse 90-day outcome, and this association increased with greater pre-morbid functional impairment. Yet, including premorbid impaired patients in the HeadPoST did not materially affect the subsequent treatment effect.

Clinical trial registration: HeadPoST is registered at http://www.ClinicalTrials.gov (NCT02162017). 
Patients with pre-morbid functional impairment, quantified by estimated scores of 2-5 on the modified Rankin scale (mRS), are generally excluded from acute stroke trials when functional recovery is used as the primary endpoint, such as in evaluations of thrombolysis, $[1,2]$ mechanical thrombectomy,[3] and intensive blood pressure (BP) lowering. ${ }^{[4-6]}$ Not only is this decision made from the viewpoint that such patients are considered less likely to benefit from acute interventions, [7] but also because of an effort to improve the precision in estimating the treatment effect using conventional dichotomous-based definitions of favorable outcome (e.g. mRS 0-1 or 0-2).[8] As a consequence, well highlighted in guidelines, there are deficiencies in the evidence-base supporting specific treatment recommendations in the 'real world', where patients with pre-morbid functional impairment are frequently encountered with acute stroke ${ }^{[9],[10]}$ and who may benefit from avoiding adverse short-[11-15] and longterm[8] outcomes. The cluster crossover design of the international Head Positioning in acute Stroke Trial (HeadPoST) included patients with acute stroke and pre-morbid functional impairment through consecutive recruitment in order to reduce selection bias and optimize implementation of the head position interventions as part of routine practice. Herein, we report analyses to quantify the impact of including patients with pre-morbid functional impairment on the Day 90 functional outcome, specifically with regard to their potential to modify the randomized treatment effect in the trial. We also outline the different patterns ('trajectory') for recovery of function according to various grades of pre-morbid levels of function.

\section{Methods}

Design

HeadPoST was a large international, multicenter, cluster-randomized, crossover, prospective, open, blinded outcome evaluated, clinical trial conducted at 114 hospitals in nine countries. 
The trial was designed to determine the comparative effectiveness of lying-flat and sitting-up head positioning (intervention), initiated soon after hospital admission in a broad range of patients with acute stroke, the full details of which are outlined elsewhere.[16, 17] Patients with a clinical diagnosis of acute ischemic stroke (AIS) or intracerebral hemorrhage (ICH) were consecutively recruited and assigned a head position according to the randomized cluster as soon as feasible after admission to hospital. Patients were encouraged to strictly maintain the randomized head position for 24 hours, but interruption was allowed for three nonconsecutive periods ( $<30$ minutes) for eating, drinking and toileting, should these not have been possible in the assigned position. HeadPoST is registered at ClinicalTrials.gov (NCT02162017).

\section{Procedures}

Key demographic and clinical characteristics were recorded at the time of enrolment, including neurological severity measured using the National Institutes of Health Stroke Scale (NIHSS) at baseline, 24 hours, and Day 7 (or earlier, on discharge from hospital). Follow-up data were collected at Day 7 (or at hospital discharge if earlier), and at Days 28 and 90, unless death occurred earlier, by independent outcome assessors blind to group allocation. Data included final diagnosis, repeated NIHSS scores, and assessment of functional impairment using the mRS. Pre-morbid mRS was assessed retrospectively through self-report by patients or an appropriate surrogate.

\section{Statistical analysis}

As an initial testing found violation of the proportional odds assumption for using $\mathrm{mRS}$ as an ordinal outcome, the primary outcome for these analyses was 'poor functional outcome', defined by mRS scores 3-6 at Day 90 post-randomization. The term early post-stroke 'additional impairment' was calculated as the difference between the pre-morbid mRS 
(estimated at baseline) and Day 7 (directly assessed; 'early $\Delta \mathrm{mRS}$ ' = Day 7 minus pre-morbid mRS scores), and evaluated as continuous and categorical measures.

Generalized linear mixed models with fixed intervention (lying-flat vs. sitting-up), and fixed period, random cluster, and random cluster-period effects were used to assess associations between early $\Delta \mathrm{mRS}$ and 90-Day functional outcomes. The model was adjusted for unbalanced baseline characteristics/prognostic variables for outcomes that included country, age, sex, baseline NIHSS scores, time from symptom onset to commencement of the head positioning intervention, and history of stroke or atrial fibrillation (AF), current smoking status, stroke subtype, and prior use of antiplatelet and anticoagulant therapy. Sensitivity analysis included further adjustment for management factors over the first 7 days, including intensive care unit admission, acute stroke unit/ward admission, and receipt of subcutaneous unfractionated heparin, antibiotic treatment, intermittent pneumatic calf compression, and physiotherapy. To assess the interaction between baseline characteristics (including premorbid $\mathrm{mRS}$ ) and association of early $\Delta \mathrm{mRS}$ and outcome, interaction terms were added to the adjusted models. Heterogeneity of the treatment effect of lying-flat and sitting-up head positioning by pre-morbid mRS was also investigated. Data are presented with adjusted odds ratios $(\mathrm{OR})$ and $95 \%$ confidence intervals $(\mathrm{CI}$, and two-sided $\mathrm{P}<0.05$ were considered statistically significant. SAS version 9.3 (SAS Institute, Cary, NC) was used for these analyses.

Latent class analysis (LCA) was used to differentiate patterns ('trajectories') of post-stroke recovery based on $\mathrm{mRS}$ scores obtained at baseline (ie pre-morbid estimates) and at Days 7 and 90. Using maximum likelihood estimation, the LCA computed the probability of each individual being assigned to a class to which they had the highest (posterior) probability of belonging,[18] and a final class was chosen to provide an optimal measure for meaningful interpretability and parsimony on Aikaike Information Classification (AIC) and Bayesian 
Information Classification (BIC) indices.[18] Differences between trajectory classes were examined by chi-square and Kruskal-Wallis for categorical and continuous variables, respectively. LCA was performed with $\mathrm{mRS}$ as a continuous measure (ordinal analysis was not feasible) using the gsem in STATA Version 9.2 (Stata Corporation, College Station, TX). A Sankey diagram for each latent class allowed description of the pattern using R statistical package (version 2.15.0, R Foundation for Statistical Computing, Vienna, Austria).

\section{Data sharing}

Individual de-identified participant data used in these analyses can be shared by formal request with protocol from any qualified investigator to the Research Office of The George Institute for Global Health, Australia.

\section{Results}

\section{Patient characteristics}

Among the HeadPoST participants (11,093 patients, mean age 68 years, 39.9\% female), 8,285 (74.7\%) had complete mRS data (pre-morbid, and Days 7 and 90). The large sample size of the study led to significant differences across most baseline characteristics between included and excluded patients (Supplemental Table SI). Of the included patients with complete data (mean age 68 years, 38.9\% female), there were 1,984 (23.9\%) with pre-morbid functional impairment (estimated mRS scores 2-5), and the overall degree of early additional impairment defined by 'early $\Delta \mathrm{mRS}$ ' mean score was 0.89 (95\%CI 0.86-0.91).

Table 1 shows that patients with greater early functional impairment (i.e. higher early $\Delta \mathrm{mRS}$ scores) were older, more often female, had earlier commencement of the head positioning intervention, and higher baseline systolic BP, heart rate and neurological impairment. They were also less likely to have had a history of stroke and to have received prior antithrombotic treatment, but more likely to have AF. 
Association of early $\triangle m R S$ with 90-Day functional outcome

Figure 1 shows the distribution of mRS scores, from pre-morbid estimates to Days 7 and 90. When analyzed as a continuous variable, every 1 point increase in early $\Delta \mathrm{mRS}$ score was associated with a $20 \%$ increase in the odds of a poor outcome (OR 1.20, 95\% CI 1.14-1.27; P $<0.0001)$. The significant positive association persisted after further adjustment for management factors (OR 1.05, 95\%CI 1.04-1.06; P <0.0001), and when early $\Delta \mathrm{mRS}$ score was analyzed as a categorical variable: compared to the reference category ( $\Delta \mathrm{mRS}$ score $\leq 0)$, the OR $(95 \% \mathrm{CI})$ across increasing $\Delta \mathrm{mRS}$ scores were: $1(0.97,0.84-1.13) ; 2(1.34,1.14-$ 1.58); and $\geq 3(2.14,1.76-2.60)$ ( $\mathrm{P}$ for linear trend 0.025) (Supplemental Figure SI). Table 2 shows that the magnitude of additional functional impairment tended to decrease with each increase in $\mathrm{mRS}$ score of pre-morbid functional impairment, becoming negative with $\mathrm{mRS}$ scores >3: adjusted mean (95\%CI) were 1.23 (1.20-1.26), 0.63 (0.58-0.69), 0.44 (0.36-0.53), $0.32(0.23-0.42),-0.08(-0.21-0.04)$ and $-0.58(-0.88--0.29)$ for $\mathrm{mRS}$ mean scores of 0 to 5 , respectively.

\section{Interaction between baseline characteristics and early $\triangle m R S$ score and outcome}

Compared with patients with good pre-morbid function (mRS scores 0 or 1 ), those with premorbid functional impairment (mRS scores 2 to 5) were significantly older, more often female, and more likely to have a history of stroke, coronary artery disease, heart failure, hypertension, AF, diabetes mellitus, and antithrombotic use, and to present with greater neurological impairment (Supplemental Table SII). However, they were less likely to develop additional early functional impairment $(\Delta \mathrm{mRS} \geq 1)$ at Day 7 (Table 2). Although overall early $\Delta \mathrm{mRS}$ mean scores were lower in patients with greater pre-morbid functional impairment, this score had prognostic significance (Table 2): OR (95\%CI) for associations of early $\Delta \mathrm{mRS}$ mean scores and Day 90 outcome were 1.70 (1.56-1.85), 1.79 (1.59-2.02), 1.86 (1.58-2.19), 2.25 (1.79-2.82), 2.43 (1.71-3.45), and $1.72(0.86-3.47)$ for pre-morbid mRS 
scores 0 to 5, respectively ( $\mathrm{P}$ for linear trend $<0.0001$ ). The association was stronger for patients recruited in China ( $\mathrm{P}$ for interaction 0.002 ) and in those who underwent reperfusion therapy (P for interaction 0.020), but was consistent across other patient subgroups defined by age, sex, NIHSS score, stroke subtype, AF, and prior use of antithrombotics (Table 2).

\section{Effect modification of pre-morbid mRS score on treatment effect}

There were no differences in the comparative effects of lying-flat and sitting-up head positioning by pre-morbid functional status (mRS scores 0-1, OR 1.08 [95\%CI 0.97-1.21] vs. mRS scores 2-5 OR 1.04 [95\%CI 0.87-1.23]) according to an ordinal shift analysis of the full range of $\mathrm{mRS}$ scores at Day $90(\mathrm{P}=0.671$ for interaction) ( Figure 1). The trial treatment effect did not change after excluding patients with pre-morbid functional impairment from analyses, nor was there any heterogeneity by level of pre-morbid functional impairment.

Among patients with AIS, reperfusion therapy was significantly associated with reduced risk of poor outcome at day 90 irrespective of premorbid mRS status $(0.55$ [0.43-0.70], and 0.62 [0.39-1.00] for premorbid mRS 0-1 and 2-5, respectively), though the association was stronger among patients with premorbid mRS 0-1 ( $\mathrm{P}=0.030$ for interaction).

\section{Patient characteristics by four patterns of the recovery trajectory}

The Sankey diagram (Figure 2) shows the temporal change in mRS scores (pre-morbid and Days 7 and 90). Table 3 outlines four patterns of recovery trajectory: to maintain stable good function (mRS mean [95\%CI] scores of 0.21 [0.19-0.22], 0.76 [0.75-0.77], and 0.68 [0.660.70] at pre-morbid, and Days 7 and 90); to make some meaningful recovery (1.21 [1.20$1.23], 1.18$ [1.16-1.20], and 0.95 [0.91-0.98]); to show decline (0.40 [0.37-0.43], 1.15 [1.121.72], 1.59 [1.58-1.61]); and to maintain disability (1.44 [1.43-1.45], 1.63 [1.62-1.65], 1.75 [1.74-1.77]). Most patients were classified as maintaining stable good functional status $(62 \%)$; the proportion in other patterns were $13 \%, 15 \%$, and $10 \%$, for making some meaningful recovery, showing decline, and maintaining disability, respectively. 
Supplemental Figure SII shows the temporal changes in mRS scores (pre-morbid, and Days 7 and 90) for each pattern.

Patients with persistent disability on follow-up had the worst baseline profile of pre-morbid function. Compared with those who showed functional decline, patients who made some meaningful recovery were younger, had less severe neurological impairment (lower NIHSS scores), but were more likely to have co-morbid cardiovascular risk factors and to have previously taken antithrombotics. Although they had a similar frequency of receiving intravenous alteplase, they were more likely to be admitted to ICU over the next 7 days compared to other patient groups.

\section{Discussion}

In this large international cohort, any deterioration in pre-morbid functional impairment experienced by patients within several days after the onset of acute stroke was associated with an increased risk of poor functional outcome at Day 90. Although this association was stronger in patients with greater pre-morbid functional impairment, who comprised approximately one quarter of the study population, their inclusion in the trial did not modify the comparative treatment effect of lying-flat versus sitting-up head positioning. Interestingly, we found that patients who made some meaningful recovery within 90 days after symptom onset, compared with those who declined, were younger and milder baseline neurological impairment but had more comorbidities.

Our results confirm findings from several European studies that noted that patients with premorbid functional impairment had a higher burden of vascular risk factors, older age, and greater neurological impairment at the onset of acute stroke.[19, 20, 8] Moreover, data from 'real world' registries, such as the Safe Implementation of Thrombolysis in Stroke-monitoring study in Eastern Europe (SITS-EAST)[21, 20] and SITS-Monitoring Study (SITS- 
MOST),[22] indicate that approximately 1 in 10 of all patients who receive intravenous alteplase $(n=904)$ have pre-morbid impairment. These data also reflect the extrapolation of randomized evidence derived from otherwise healthy populations/people into clinical practice to frailer, disabled patients, who under-represented in acute stroke trials.

Despite strong evidence that intravenous alteplase improves the chances of recovery after AIS in a time dependent manner, irrespective of age or neurological severity,[23] there is uncertainty over the net balance of benefits over risks of this treatment in those with preexisting functional impairment. ${ }^{[9],[10]}$ Our analyses provide some justification for including these patients in research and practice. First, reperfusion therapy improved recovery at day 90 irrespective of pre-existing functional impairment, though the magnitude of the association was stronger among those with premorbid mRS 0-1. Second, we have shown that the degree of additional stroke-related functional impairment had a greater impact on long-term functional outcome in those with pre-morbid functional impairment compared to those without. Interventions with the potential to minimize additional functional impairment could translate into longer benefits in this group. Third, in AIS patients who receive reperfusion therapy, those with higher levels of early additional functional impairment have poor outcomes, but where the addition of functional impairment was associated with factors that reflect the underlying cause of stroke (age, sex, neurological severity, presence of AF), not the level of pre-morbid functional status. Finally, the inclusion of patients with pre-morbid functional impairment did not affect either the point estimate or precision of the treatment effect in the HeadPoST trial.

We found that patients who made some meaningful recovery were younger and had less severe initial neurological impairment despite a greater number of comorbid conditions compared with those who had shown decline. This is consistent with findings from literature, such as the study by Faircloth et al[24, 25] who identified three factors that might mitigate 
against the disruptive impact of stroke: age, comorbidities and preexisting knowledge of stroke. In regard to the latter factor, the impact of the stroke may be lessened if the illness can be viewed as a natural part of growing older and where people are already managing existing comorbidities. We recognize, however, that our analyses were restricted to the physical and functional aspects of rehabilitation and that future studies are needed to understand the dynamic process of recovery that is shaped by the interaction of physical, emotional, and social domains.[26, 27]

A key strength of these analyses is their generalizability: despite being derived from a clinical trial of patients with acute stroke of predominantly mild to moderate neurological severity (median NIHSS score 4), the recruitment strategy was undertaken across a range of clinical settings to provide data on a broad range of stroke phenotypes. However, as in any observational study, there is the potential for confounding from incomplete adjustment, in particular those related to management confounders in the first 7 days, and recall bias from the assessment of pre-morbid mRS through self-report. In addition, there were $25 \%$ missing data which may also introduced bias as reflected in the proportion of patients with pre-morbid impairment (mRS 2-5) being lower in the excluded than included group.

In summary, any additional functional impairment experienced early after the onset of acute stroke was associated with worse Day-90 outcomes, and this association was stronger in patients with greater pre-existing functional impairment. Even so, patients with pre-morbid functional impairment may gain from treatments that limit further deterioration in function. Our analyses, therefore, provide some support for the enrolment of patients with pre-morbid disability into acute stroke trials. 


\section{Author contributions}

XW and TM wrote the first draft of the report, XW and MO contributed to data analysis. LB, KS and CSA supervised the analyses. XW and CSA contributed to the concept and rationale for the study, and wrote the report. Others contributed to data collection and provided comments on data interpretation and the report.

\section{Statement of ethics:}

The appropriate ethics committee at each participating centre approved the study protocol. A senior executive officer at each centre acted as a 'guardian' and provided institutional consent for this low-risk intervention to be implemented as part of routine nursing care in each cluster. Written informed consent was sought from all patients or approved surrogates for ongoing assessments and data collection.

\section{Funding sources:}

The HeadPoST trial is supported by a research grant (1066966) from the National Health and Medical Research Council (NHMRC) of Australia. XW holds National Heart Foundation of Australia post-doctoral fellowship, investigator development grant from New South Wales Health commission, Australia, and investigator grant from NHMRC of Australia. TJM holds a British Heart Foundation clinical research training fellowship. ECS holds a postdoctoral fellowship from the South Eastern Norway Regional Health Authority. TGR is Senior Investigators of the National Institute for Health Research (NIHR) of the UK. CSA holds a Senior Investigator Fellowship of the NHMRC of Australia. All authors had full access to data for this study.

These agencies had no role in the design of the trial protocol, in the collection, analysis, or interpretation of the trial data, or in the writing of the manuscript.

\section{Disclosure}


Dr. Hackett, receiving travel support from Boehringer Ingelheim; Dr. Robinson, receiving lecture fees and advisory panel fees from Bayer, lecture fees from Boehringer Ingelheim, and advisory panel fees from Daiichi Sankyo; Dr. Lavados, receiving support to attend meetings from Boehringer Ingelheim, grant support, fees for serving as country leader for the New Approach Rivaroxaban Inhibition of Factor Xa in a Global Trial versus ASA to Prevent Embolism in Embolic Stroke of Undetermined Source (NAVIGATE ESUS) trial, and travel support from Bayer, grant support and fees for serving as country leader for the Acute Stroke or Transient Ischaemic Attack Treated with Aspirin or Ticagrelor and Patient Outcomes (SOCRATES) trial from AstraZeneca, and grant support from Comisión Nacional de Investigación Científica y Tecnológica (CONICYT); Dr. Olavarría, receiving grant support from the George Institute for Global Health; Dr. Muñoz Venturelli, receiving grant support from Clínica Alemana de Santiago and support from the George Institute for Global Health, paid to the author's institution; Dr. Arima, receiving lecture fees from Takeda, Daiichi Sankyo, Astellas, and Aska Pharmaceuticals; Dr. Pontes-Neto, receiving lecture fees from Boehringer Ingelheim and Medtronic; Dr. Anderson reports receiving advisory board fees from Medtronic and AstraZeneca and lecture fees and travel support from Takeda China and Boehringer Ingelheim; Dr Sandset reports receiving speaking fees from Bayer and Novartis; and No other potential conflict of interest relevant to this article was reported. 


\section{References}

1. Emberson J, Lees KR, Lyden P, Blackwell L, Albers G, Bluhmki E, et al. Effect of treatment delay, age, and stroke severity on the effects of intravenous thrombolysis with alteplase for acute ischaemic stroke: a meta-analysis of individual patient data from randomised trials. Lancet. 2014 Nov 29;384(9958):1929-35.

2. Anderson CS, Robinson T, Lindley RI, Arima H, Lavados PM, Lee T-H, et al. Low-Dose versus Standard-Dose Intravenous Alteplase in Acute Ischemic Stroke. 2016;374(24):2313-23.

3. Goyal M, Menon BK, van Zwam WH, Dippel DW, Mitchell PJ, Demchuk AM, et al. Endovascular thrombectomy after large-vessel ischaemic stroke: a meta-analysis of individual patient data from five randomised trials. Lancet. 2016 Apr 23;387(10029):1723-31.

4. Anderson CS, Huang Y, Wang JG, Arima H, Neal B, Peng B, et al. Intensive blood pressure reduction in acute cerebral haemorrhage trial (INTERACT): a randomised pilot trial. Lancet Neurol. 2008 May;7(5):391-9.

5. Delcourt C, Huang Y, Wang J, Heeley E, Lindley R, Stapf C, et al. The second (main) phase of an open, randomised, multicentre study to investigate the effectiveness of an intensive blood pressure reduction in acute cerebral haemorrhage trial (INTERACT2). Int J Stroke. 2010 Apr;5(2):110-6.

6. Anderson CS, Heeley E, Huang Y, Wang J, Stapf C, Delcourt C, et al. Rapid blood-pressure lowering in patients with acute intracerebral hemorrhage. N Engl J Med. 2013 Jun 20;368(25):2355-65.

7. Ganesh A, Luengo-Fernandez R, Pendlebury ST, Rothwell PM. Long-Term Consequences of Worsened Poststroke Status in Patients With Premorbid Disability. 2018;49(10):2430-36.

8. Ganesh A, Luengo-Fernandez R, Pendlebury ST, Rothwell PM. Long-Term Consequences of Worsened Poststroke Status in Patients With Premorbid Disability. Stroke. 2018 Oct;49(10):2430-36.

9. Fiehler J, Cognard C, Gallitelli M, Jansen O, Kobayashi A, Mattle HP, et al. European Recommendations on Organisation of Interventional Care in Acute Stroke (EROICAS). Int J Stroke. 2016 Aug;11(6):701-16.

10. Powers WJ, Rabinstein AA, Ackerson T, Adeoye OM, Bambakidis NC, Becker K, et al. 2018 Guidelines for the Early Management of Patients With Acute Ischemic Stroke: A Guideline for Healthcare Professionals From the American Heart Association/American Stroke Association. Stroke. 2018 Mar;49(3):e46-e110.

11. Kwok CS, Clark A, Ford GA, Durairaj R, Dixit AK, Davis J, et al. Association between prestroke disability and inpatient mortality and length of acute hospital stay after acute stroke. J Am Geriatr Soc. 2012 Apr;60(4):726-32.

12. O'Donnell MJ, Fang J, D'Uva C, Saposnik G, Gould L, McGrath E, et al. The PLAN score: a bedside prediction rule for death and severe disability following acute ischemic stroke. Arch Intern Med. 2012 Nov 12;172(20):1548-56.

13. Karlinski M, Kobayashi A, Czlonkowska A, Mikulik R, Vaclavik D, Brozman M, et al. Role of Preexisting Disability in Patients Treated With Intravenous Thrombolysis for Ischemic Stroke. 2014;45(3):770-75.

14. Abdul-Rahim AH, Quinn TJ, Alder S, Clark AB, Musgrave SD, Langhorne $P$, et al. Derivation and Validation of a Novel Prognostic Scale (Modified-Stroke Subtype, Oxfordshire Community Stroke Project Classification, Age, and Prestroke Modified Rankin) to Predict Early Mortality in Acute Stroke. Stroke. 2016 Jan;47(1):74-9.

15. Quinn TJ, Taylor-Rowan M, Coyte A, Clark AB, Musgrave SD, Metcalf AK, et al. Pre-Stroke Modified Rankin Scale: Evaluation of Validity, Prognostic Accuracy, and Association with Treatment. Front Neurol. 2017;8:275. 
16. Munoz-Venturelli $\mathrm{P}$, Arima $\mathrm{H}$, Lavados $\mathrm{P}$, Brunser A, Peng B, Cui L, et al. Head Position in Stroke Trial (HeadPoST)--sitting-up vs lying-flat positioning of patients with acute stroke: study protocol for a cluster randomised controlled trial. Trials. 2015 Jun 5;16:256.

17. Anderson CS, Arima H, Lavados P, Billot L, Hackett ML, Olavarria VV, et al. ClusterRandomized, Crossover Trial of Head Positioning in Acute Stroke. N Engl J Med. 2017 Jun 22;376(25):2437-47.

18. Lanza ST, Rhoades BL. Latent class analysis: an alternative perspective on subgroup analysis in prevention and treatment. Prev Sci. 2013;14(2):157-68.

19. Foell RB, Silver B, Merino JG, Wong EH, Demaerschalk BM, Poncha F, et al. Effects of thrombolysis for acute stroke in patients with pre-existing disability. CMAJ. 2003 Aug 5;169(3):193-7.

20. Karlinski M, Kobayashi A, Czlonkowska A, Mikulik R, Vaclavik D, Brozman M, et al. Role of preexisting disability in patients treated with intravenous thrombolysis for ischemic stroke. Stroke. 2014 Mar;45(3):770-5.

21. Karlinski M, Kobayashi A, Mikulik R, Sanak D, Wahlgren N, Czlonkowska A, et al. Intravenous alteplase in ischemic stroke patients not fully adhering to the current drug license in Central and Eastern Europe. Int J Stroke. 2012 Dec;7(8):615-22.

22. Wahlgren N, Ahmed N, Davalos A, Ford GA, Grond M, Hacke W, et al. Thrombolysis with alteplase for acute ischaemic stroke in the Safe Implementation of Thrombolysis in StrokeMonitoring Study (SITS-MOST): an observational study. Lancet. 2007 Jan 27;369(9558):27582.

23. Emberson J, Lees KR, Lyden P, Blackwell L, Albers G, Bluhmki E, et al. Effect of treatment delay, age, and stroke severity on the effects of intravenous thrombolysis with alteplase for acute ischaemic stroke: a meta-analysis of individual patient data from randomised trials. The Lancet. 2014;384(9958):1929-35.

24. Furness PJ, Garrud P. Adaptation after facial surgery: using the diary as a research tool. Qualitative health research. 2010 Feb;20(2):262-72.

25. Hawkins RJ, Jowett A, Godfrey M, Mellish K, Young J, Farrin A, et al. Poststroke Trajectories: The Process of Recovery Over the Longer Term Following Stroke. Glob Qual Nurs Res. 2017 Jan-Dec;4:2333393617730209.

26. Dowswell G, Lawler J, Dowswell T, Young J, Forster A, Hearn J. Investigating recovery from stroke: a qualitative study. Journal of clinical nursing. $2000 \mathrm{Jul} ; 9(4): 507-15$.

27. Kendall E, Catalano T, Kuipers P, Posner N, Buys N, Charker J. Recovery following stroke: the role of self-management education. Social science \& medicine (1982). 2007 Feb;64(3):73546. 
Figure legend

Figure 1 Randomized treatment effect of lying-flat versus sitting-up head positioning on functional outcome according to $\mathrm{mRS}$ score at Day 90, by premorbid mRS score

Footnote: CI denotes confidence interval, OR odds ratio.

Scores on the mRS range from 0 to 6 , with 0 indicating no symptoms, 1 symptoms without clinical significant disability, 2 slight disability, 3 moderate disability, 4 moderately severe disability, 5 severe disability, and 6 death.

Figure 2. Sankey diagram of the distribution of disability, pre-morbid and at Days 7 and 90, on the modified Rankin scale (mRS)

Footnote: mRS denotes modified Rankin scale

Each color represents different mRS scores; the 3 horizontal positions indicate pre-morbid, and Days 7 and 90, time points; the vertical ordering shows mRS scores 0 to 6 .

Changes of pre-morbid, and Days 7 and 90 mRS scores are shown in the table below:

\begin{tabular}{|c|c|c|c|c|c|c|c|c|c|c|c|c|c|c|}
\hline \multirow{2}{*}{$\begin{array}{l}\text { Premorbid } \\
\text { mRS }(n, \%)\end{array}$} & \multicolumn{7}{|c|}{ mRS at Day $7(\%)$} & \multicolumn{7}{|c|}{ mRS at Day $90(\%)$} \\
\hline & 0 & 1 & 2 & 3 & 4 & 5 & 6 & 0 & 1 & 2 & 3 & 4 & 5 & 6 \\
\hline $0(4690,56.6)$ & 27.4 & 35.7 & 23.2 & 13.7 & - & - & - & 24.7 & 44.7 & 10.1 & 12.4 & 4.4 & 2.3 & 1.5 \\
\hline $1(1611,19.4)$ & 11.1 & 48.0 & 18.6 & 11.3 & 11.1 & - & - & 18.0 & 40.7 & 8.8 & 15.9 & 7.9 & 4.6 & 4.2 \\
\hline $2(981,11.8)$ & 4.7 & 18.5 & 38.0 & 15.4 & 13.5 & 10.0 & - & 12.7 & 31.9 & 7.9 & 19.1 & 12.7 & 7.2 & 8.5 \\
\hline $3(620,7.5)$ & 0.8 & 5.7 & 14.8 & 37.9 & 21.9 & 17.6 & 1.3 & 3.9 & 21.6 & 5.5 & 22.4 & 15.2 & 12.6 & 18.9 \\
\hline $4(306,3.7)$ & 1.6 & 2.9 & 5.6 & 12.4 & 50.0 & 24.2 & 3.3 & 4.6 & 11.1 & 4.9 & 14.4 & 20.3 & 19.0 & 25.8 \\
\hline $5(77,0.9)$ & 3.9 & 2.6 & 2.6 & 3.9 & 15.6 & 68.8 & 2.6 & 1.3 & 3.9 & 3.9 & 15.6 & 16.9 & 28.6 & 29.9 \\
\hline Total & 18.4 & 32.3 & 22.6 & 15.1 & 7.4 & 4.0 & 0.2 & 19.5 & 39.1 & 9.0 & 14.7 & 7.6 & 4.9 & 5.3 \\
\hline
\end{tabular}

